\title{
The detection of communicative signals directed at the self in infant prefrontal cortex
}

\author{
Tobias Grossmann ${ }^{1,2 * t}$, Eugenio Parise ${ }^{2,3 * t}$ and Angela D. Friederici ${ }^{2}$ \\ 1 Centre for Brain and Cognitive Development, Birkbeck, University of London, London, UK \\ 2 Department of Neuropsychology, Max Planck Institute for Human Cognitive and Brain Sciences, Leipzig, Germany \\ ${ }^{3}$ Cognitive Development Center, Central European University, Budapest, Hungary
}

\author{
Edited by: \\ Harold Bekkering, University of \\ Nijmegen, Netherlands \\ Reviewed by: \\ Sharon Fox, Massachusetts Institute of \\ Technology, USA \\ *Correspondence: \\ Tobias Grossmann, Centre for Brain \\ and Cognitive Development, Birkbeck, \\ University of London, Malet Street, \\ London WC1E 7HX, UK. \\ e-mail: t.grossmann@bbk.ac.uk; \\ Eugenio Parise, Cognitive \\ Development Center, Central \\ European University, Hattyú u. 14, 1015 \\ Budapest, Hungary. \\ e-mail: eugenioparise@tiscali.it \\ tTobias Grossmann and Eugenio Parise \\ contributed equally to this paper.
}

A precondition for successful communication between people is the detection of signals indicating the intention to communicate, such as eye contact or calling a person's name. In adults, establishing communication by eye contact or calling a person's name results in overlapping activity in right prefrontal cortex, suggesting that, regardless of modality, the intention to communicate is detected by the same brain region. We measured prefrontal cortex responses in 5-month-olds using near-infrared spectroscopy (NIRS) to examine the neural basis of detecting communicative signals across modalities in early development. Infants watched human faces that either signaled eye contact or directed their gaze away from the infant, and they also listened to voices that addressed them with their own name or another name. The results revealed that infants recruit adjacent but non-overlapping regions in the left dorsal prefrontal cortex when they process eye contact and own name. Moreover, infants that responded sensitively to eye contact in the one prefrontal region were also more likely to respond sensitively to their own name in the adjacent prefrontal region as revealed in a correlation analysis, suggesting that responding to communicative signals in these two regions might be functionally related. These NIRS results suggest that infants selectively process and attend to communicative signals directed at them. However, unlike adults, infants do not seem to recruit a common prefrontal region when processing communicative signals of different modalities. The implications of these findings for our understanding of infants' developing communicative abilities are discussed.

Keywords: eye contact, name, communication, intention, prefrontal cortex, infancy

\section{INTRODUCTION}

The ability to detect whether one is being addressed is of critical importance to successfully communicate with others. Before decoding the message that is conveyed by another person, one must pick up on signals that indicate that the message is in fact directed at the self (i.e., self-referential). The most common way to initiate intentional communication is by using so-called ostensive signals such as calling someone's name and looking directly at someone to establish eye contact. Although these two ostensive signals differ in modality and most low-level perceptual features, they both serve to make the intention to communicate manifest to the receiver (Sperber and Wilson, 1995; Csibra, 2010).

Adults are highly sensitive to eye contact and name cues, which powerfully grab their attention even when they are engaged in cognitively demanding tasks (Moray, 1959; von Grünau and Anston, 1995). Critically, it has been argued that mentalizing, the ability to attribute mental states such as intentions to others (Frith and Frith, 1999, 2006), is involved in understanding communication already at the stage at which a sender initiates communication through eye contact or calling someone's name. Accordingly, Kampe et al. (2003) predicted that brain regions implicated in mentalizing would be engaged when detecting communicative signals as referring to the self. Indeed, using functional resonance imaging (fMRI), Kampe et al. (2003) have shown that, in adults, calling a person's name or making eye contact activate common brain regions that are implicated in attributing mental states to others (Frith and Frith, 1999, 2006). More specifically, the brain regions adults employ in processing these communicative signals are localized in the right medial prefrontal cortex and the left temporal pole (Kampe et al., 2003). These findings suggest that, in adults, the communicative intention of another person directed toward oneself is detected by the same brain region, regardless of modality. This process occurs without deliberate attention (participants were asked to detect faces with eyes closed rather than direct gaze or to detect last names rather than their own name) and it also does not depend on subsequent interaction between participants. More support for the view that mentalizing is critical for understanding ostensive communication cues comes from individuals with autism who have been shown to be impaired in their mentalizing skills and also have severe difficulties in recognizing when they are being addressed (Baron-Cohen, 2000). Indeed, lack of orienting to name and eye contact is perhaps one of the earliest signs of autism identifiable in childhood (Osterling et al., 2002).

Taken together, this poses the important ontogenetic question of whether and when typically developing infants are sensitive to eye contact and own name as ostensive signals of communicative intent and more specifically, whether infants would engage brain regions (and thus cognitive processes) similar to adults when they respond to ostensive signals across modalities. Findings as to whether young infants already engage in adult-like brain processes 
associated with mentalizing when they interpret communicative signals can inform theories of early social-cognitive development and may add to recent work that has shown that infants understand certain kinds of intentions and other mental states surprisingly early in development (Woodward, 2009; Baillargeon et al., 2010). The current study will deal with these questions. From previous work it is known that infants are sensitive to eye contact from birth (Farroni et al., 2002, 2005), suggesting that eye contact is a potentially hard-wired and pre-specified cue detected without or at least with only very little prior experience. First names on the other hand are arbitrary auditory signals chosen by the parents that must be learned by the infant. Sensitivity to own name emerges around 4.5 months of age when infants have been shown to first orient to their name (Mandel et al., 1995).

In the current study, we measured prefrontal cortex responses in 5-month-old infants using near-infrared spectroscopy (NIRS) permitting spatial localization of brain activation by measuring hemodynamic responses to examine the neural basis and developmental origins of detecting ostensive communicative signals across modalities (see Lloyd-Fox et al., 2010, for a detailed review of NIRS and its use with infants). Other neuroimaging techniques that are well established in adults are limited in their use with infants because of methodological concerns. For example, positron emission tomography (PET) exposes participants to radioisotopes, and $\mathrm{fMRI}$ requires the participant to remain very still and exposes them to a noisy environment. Although both PET and fMRI have been used with infants, this work is restricted to the study of sleeping, sedated or very young infants. NIRS is better suited for infant research because it can accommodate a good degree of movement from the infants, enabling them to sit upright on their parent's lap and behave relatively freely while watching or listening to certain stimuli. In addition, unlike PET and fMRI, NIRS systems are portable. Finally, despite its inferior spatial resolution, NIRS, like fMRI, measures localized patterns of hemodynamic responses, thus allowing for a comparison of infant NIRS data with adult fMRI data (see Strangman et al., 2002, for evidence of a strong correlation between hemodynamic responses measured with fMRI and NIRS).

We studied 5-month-old infants because, as mentioned above, at this age they have been shown to be sensitive to eye contact as well as name cues. Moreover, we focused on prefrontal cortical responses since this is one of the parts of the brain that showed common activation to eye contact and name cues in adults (Kampe, et al., 2003), and previous infant NIRS studies have successfully obtained specialized prefrontal cortex responses in social tasks (Grossmann, et al., 2008; Grossmann and Johnson, 2010). Infants watched human faces that either signaled eye contact or directed their gaze away from the infant, and they also listened to voices that addressed them with their own name or with the name of another infant.

\section{MATERIALS AND METHODS PARTICIPANTS}

Twenty 5-month-old infants were included in the final sample (11 girls, range 139-166 days, $M=151.8$ days). Nine additional infants were tested but not included in the final sample because of fussiness $(N=4)$, too many motion artifacts $(N=3)$, technical problems or experimenter errors $(N=2)$. All infants contributed a total of four trials for each experimental condition. The required minimum number of artifact-free trials per condition was 2 (see section Data Acquisition and Analysis for artifact treatment). Please note that an attrition rate at this level is within the normal range for an infant NIRS study (Lloyd-Fox et al., 2010). All infants were born full-term (37-42 weeks gestation) and with normal birthweight (>2500 g). All parents gave informed consent before the study.

\section{STIMULI AND PROCEDURE}

Visual and auditory stimuli were presented. Visual stimuli were static pictures of a smiling face depicting mutual or averted gaze (left or right). The faces subtended a visual angle of $22^{\circ} \times 17^{\circ}$ at a viewing distance of $60 \mathrm{~cm}$. Auditory stimuli were audio files $(\mathrm{SPL}=70 \mathrm{~dB})$. Each infant heard her own name and a stranger's name, matched for syllable number and always differing from the infant's name in its first phoneme. All names were spoken in infant-directed speech by a female voice. Infants sat on their parent's lap while watching the stimuli on a computer monitor within an acoustically shielded, dimly lit room. The experiment consisted of two sessions balanced across participants: a visual and an auditory session. Each session consisted of eight trials, four per condition. Each trial consisted of three presentations of the stimulus, each presentation lasting for $1000 \mathrm{~ms}$ for the pictures and an average of $790 \mathrm{~ms}$ for the names (ranging from 394 to $1228 \mathrm{~ms}$ ). Own name and control name did not differ in their duration. A star appeared on the center of the screen for $300 \mathrm{~ms}$ before and after each stimulus. The screen was black when names were presented. The two experimental conditions within each session were randomly distributed, with no more than two trials of the same condition occurring in a row. The inter-trial interval varied randomly between 10 and $12 \mathrm{~s}$. Non-social moving visual stimuli were presented during the inter-trial interval to keep infants' attention.

\section{DATA ACOUISITION AND ANALYSIS}

The NIRS method relies on the optical determination of changes in hemoglobin concentrations in cerebral cortex which result from increased regional cerebral blood flow. Prefrontal cortex activations were measured using a Hitachi ETG-4000 NIRS system. Two wavelengths were set at 695 and $830 \mathrm{~nm}$ for all recording channels. Two custom-built arrays consisting of nine optodes (five sources, four detectors) in a 12-channel (source-detector pairs) arrangement with an inter-optode separation of $25 \mathrm{~mm}$ were placed over the frontal lobe on each hemisphere using an Easycap (www.easycap. de). NIRS data were continuously sampled at $10 \mathrm{~Hz}$. Our current measurement technique did not provide us with information about the depth of the activation (see Blasi et al., 2007, for NIRS methodology that allows for the measurement of depth-dependent hemodynamic responses in infants). After calculation of the oxyHb concentration changes, pulse-related signal changes and overall trends were eliminated by low-pass filtering (Butterworth, fifth order, lower cutoff $0.5 \mathrm{~Hz}$ ). Movement artifacts were corrected by an established procedure (see Grossmann et al., 2010), which allows marking of artifacts and then padding the contaminated data segments by linear interpolation. After visual inspection of the time course of the concentration changes a time window around the peak of the hemodynamic response (between 5 and $7 \mathrm{~s}$ after stimulus onset) was chosen for statistical analysis. Cortical responses were 
assessed by comparing average concentration changes (oxyHb) within trials for this time window between the two experimental conditions. Paired $t$-test were used in order to compare between conditions within each modality: eye contact versus averted gaze in the visual modality and infant's own name versus stranger's name in the auditory modality. Please note that similar to previous NIRS studies (see Lloyd-Fox et al., 2010) p-values were not corrected for multiple comparisons.

\section{RESULTS}

Our analysis of 5-month-old infants' prefrontal brain responses revealed two adjacent regions in the left hemisphere sensitive to the communicative signals conveyed to the infant (see Figure 1). Specifically, a left dorsal prefrontal region showed sensitivity to infants' own name as indexed by a significantly increased oxyHb concentration (average of concentration change between 5 and $7 \mathrm{~s}$ after stimulus onset) when own name was compared to the control name (channel 9: $t[19]=3.060, p=0.006$ ), while an adjacent left dorsal prefrontal region responded sensitively to eye contact cues as indexed by a significantly increased oxyHb concentration when eye contact is compared to averted gaze (channel 6: $t[19]=2.291, p=0.034$ ). These results were confirmed by non-parametric tests (Wilcoxon's $z=2.053, p=0.040$ for the visual modality [channel 6, 13 out of 20 infants showed the effect] and Wilcoxon's $z=2.539, p=0.011$, for the auditory modality [channel 9, 14 out of 20 infants showed the effect]).

Furthermore, the prefrontal cortex responses to eye contact (mutual gaze-averted gaze) and name (own name-control name) were significantly positively correlated $(r=0.446, p=0.049)$, indicating that infants who showed a sensitive prefrontal cortex response to eye contact were more likely to also show a sensitive prefrontal cortex response to their own name (see Figure 2). There were no significant correlations between channels 6 and 9 when calculating the overall association of the responses for the four individual conditions (mutual gaze, averted gaze, own name, and other name), suggesting that the correlation reported above was specific to the contrasts calculated (mutual gaze-averted gaze and own name-control name) and not due to a general association of hemodynamic responses between channels. This also renders it unlikely that the NIRS signals measured from channels 6 and 9 were sampled from a common vascular supply. Our analysis revealed no significant effects of the factors gender and presentation order (auditory first versus visual first) for channels 6 and 9. There were no significant correlations between any of the other channels.

An additional analysis performed after close inspection of the time courses at these channels showed that there was an earlier effect (1-3 s after stimulus onset) of eye contact at channel 6 (increased oxyHb concentration when eye contact was compared to averted gaze, $t[19]=2.176, p=0.042$ (Wilcoxon's $z=2.613, p=0.009 ; 14$ out of 20 infants showed the effect), whereas no such early effect of name was observed during auditory stimulation (see Figure 1).

\section{DISCUSSION}

We examined whether 5-month-old infants are sensitive to eye contact and their own name as ostensive signals of communicative intent regardless of modality by measuring prefrontal cortex responses using NIRS. The results revealed two adjacent regions
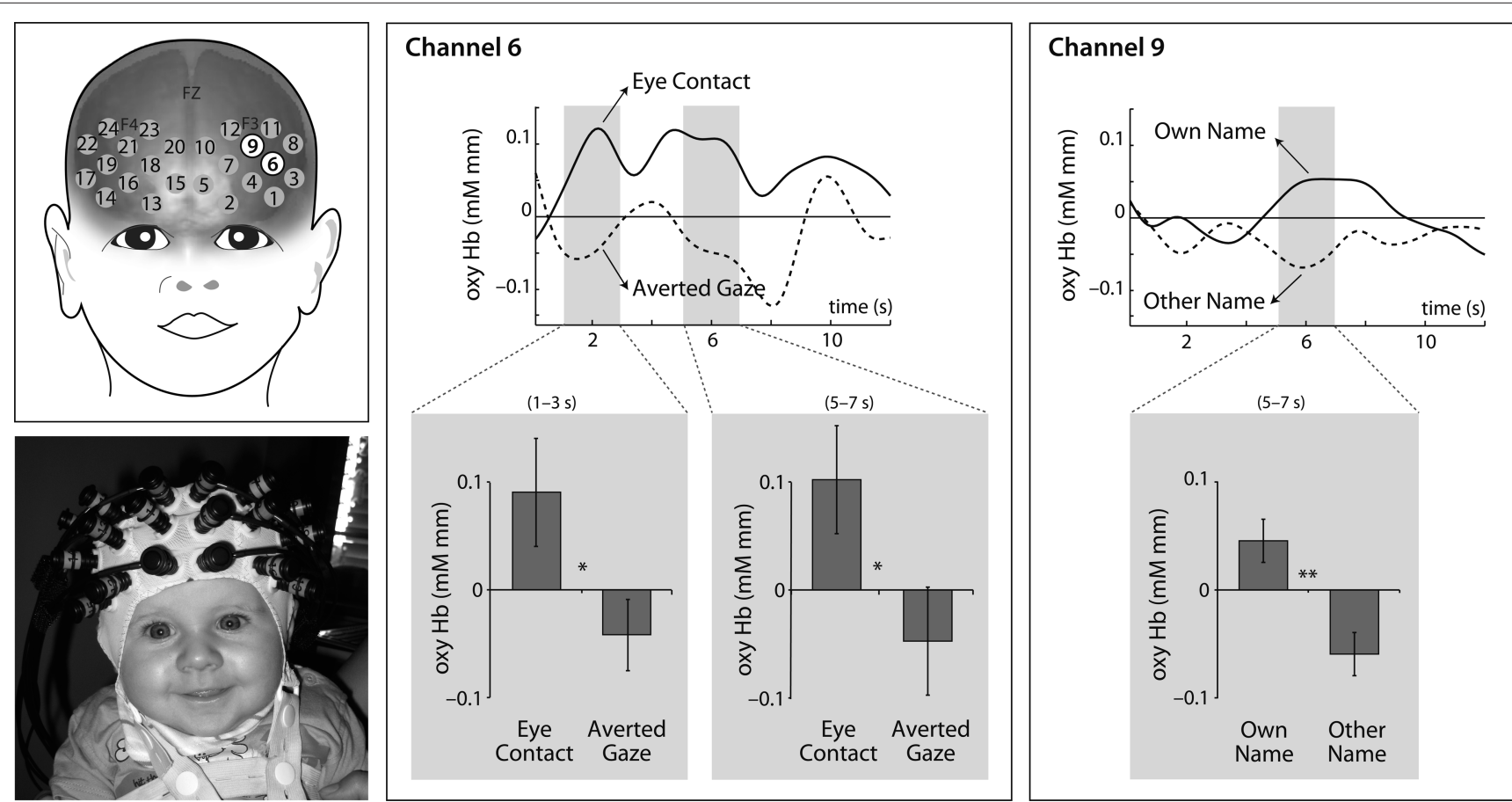

represent the time windows during which significant differences between conditions were observed. The bottom row shows bar charts with the average oxyHb change ( \pm SE) observed during the time windows marked in the

FIGURE 1 | Five-month-old infants' prefrontal cortex responses to eye contact (channel 6) and own name (channel 9). Time courses (with 0 indicating the stimulus onset) of the hemodynamic response for these two channels are shown in the top row. The gray windows in the time courses top row. 


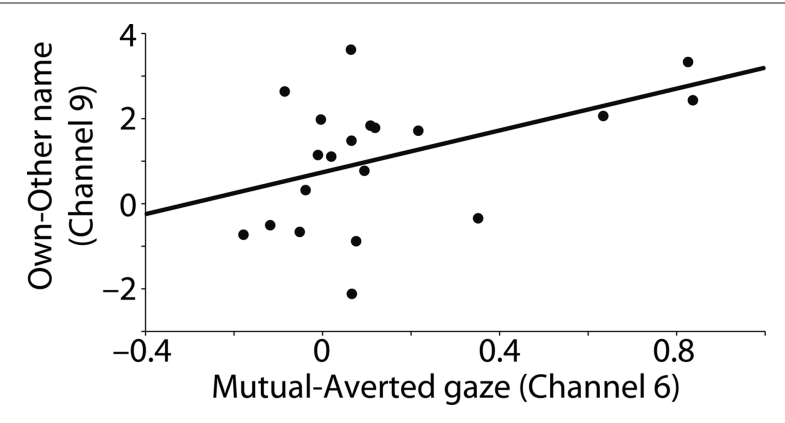

FIGURE 2 | Correlation between prefrontal cortex responses to eye contact (channel 6: difference in oxyHb [mutual-averted gaze] in $\mathrm{mM} \mathrm{mm}$ ) and own name (channel 9: difference in oxyHb [own-other name] in $\mathbf{m M ~} \mathbf{m m}$ ).

in the left prefrontal cortex that are either sensitive to the communicative signals conveyed to the infant through face or voice. Specifically, a left dorsal prefrontal region showed sensitivity to infants' own name, while an adjacent left dorsal prefrontal region responded sensitively to eye contact cues. Thus, these NIRS results suggest that 5-month-old infants selectively process and attend to communicative signals directed at them as reflected in their specific prefrontal cortex responses within each modality.

Interestingly, unlike adults, 5-month-old infants do not seem to recruit a common right prefrontal region when processing communicative signals across modalities. However, infants showed a correlation in the activation of the two prefrontal regions involved. Infants who responded sensitively to eye contact in one of the left prefrontal regions were more likely to respond sensitively to their own name in the adjacent region as revealed in a correlation analysis, suggesting that responding to ostensive communicative signals in these two regions might be functionally correlated. This is perhaps an important developmental precursor for a later integration into a more abstract and modality-independent representation of communicative signals directed at the self.

It is important to further discuss the question of how these findings using NIRS with infants relate to the findings using fMRI with adults in order to understand the development of processing communicative signals. While infants in the current study showed sensitive responding to eye contact and own name in the left prefrontal cortex, the effect in adults for the same contrast revealed activation in the right prefrontal cortex (Kampe, et al., 2003). The difference in the lateralization of the activation patterns between infants and adults suggests different underlying processes that might be due to development, differences in the stimulus material used, or both. In contrast to the adult work, we used smiling faces and infant-directed voices that are both characterized by positive affect. The presentation of positive affect in face and voice directed at the infant may have induced the motivation to approach, which, according to prior infant and adult work, is reflected in left prefrontal activation (Davidson and Fox, 1982; Fox, 1991; Harmon-Jones, 2003). This raises the possibility that infants responded to the positive affective signals directed at them with the motivation to approach rather than with mentalizing like adults do in response to communicative signals. This account does not undermine infants' sensitivity to eye contact and own name but it may point to a developmental difference between infants and adults in the interpretation of these communicative signals.

However, it should be noted that this is only one potential account for the observed developmental difference. Future work should more directly address the question of what kind of psychological process can best account for young infants' left prefrontal response patterns during these kinds of communicative scenarios. For example, according to the motivational account put forward above it would be predicted that by using faces and voices expressing anger toward the infant one should observe a different pattern of lateralization in their prefrontal cortex responses since prior work suggests that perceiving aversive emotions results in right prefrontal activation associated with a motivation to withdraw (Davidson and Fox, 1982; Fox, 1991; Harmon-Jones, 2003).

Another issue that deserves attention when discussing the current findings is the selection of stimuli used. Specifically, in order to make it comparable to prior adult work (Kampe et al., 2003) we used static faces in the visual part of the study. From previous infant work it is known that using dynamic rather than static representation of faces has been shown to increase infants' attention and cortical responses (Grossmann et al. 2008; Grossmann and Farroni, 2009). Although we obtained significant effects in both the face and name context, the effect (as indicated by the $t$-values) appeared to be somewhat weaker in the face than in the name context. Therefore, presenting infants with a dynamic face that shifts gaze and engages them in eye contact rather than a static face in future studies may be a clearer and more ecologically valid indicator of the intention to communicate with the infant.

Our NIRS results further revealed that, while eye contact cues and name cues resulted in non-overlapping prefrontal cortex responses between 5 and $7 \mathrm{~s}$ after stimulus onset, only eye contact evoked an early increase in the hemodynamic response between 1 and $3 \mathrm{~s}$ after stimulus onset. This finding suggests that infants recruit particularly fast prefrontal brain processes for the detection of eye contact. This rapid prefrontal response may be related to the fact that eye contact is an especially powerful social cue that infants are sensitive to from birth, and it supports the hypothesis of specialized brain mechanisms that guarantee a fast detection of eye contact (Senju and Johnson, 2009). In particular, a fast subcortical pathway involving the superior colliculus and the pulvinar has been hypothesized to convey information to the cerebral cortex including prefrontal areas (Johnson, 2005). Processes of eye contact detection stand in contrast to the detection of the own name, which is an arbitrary signal to the self that has to be learned by the infant. Our data show that infants as young as 5 months of age are sensitive to this acquired ostensive signal and selectively recruit a specific region of the left prefrontal cortex. Thus, the prefrontal cortex also plays a role in integrating learned information into the infants communication comprehension repertoire.

In summary, the current data have provided new insights into young infants' processing and understanding of communicative signals directed at them. The sensitivity to eye contact and own name demonstrated in selective prefrontal cortex responses may serve as a pivotal building block that helps infants to enter the world of human communication. We have identified developmental precursors of the brain processes that are involved in initiating communication. However, the exact nature of the representation reflected in infants' 
selective prefrontal cortex responses remains unclear. In particular, the present data leave open whether infants already engage adult-like mentalizing processes. This question has been of great interest to developmental and cognitive psychologists in recent years (see Woodward, 2009; Baillargeon et al., 2010) and certainly needs further examination in future studies. The current study has shown that a neuroscience perspective can provide additional insights into preverbal infants' developing social-cognitive and communicative abilities.

\section{REFERENCES}

Baillargeon, R., Scott, R. M., and He, Z. (2010). False-belief understanding in infants. Trends Cogn. Sci. 14, 110-118.

Baron-Cohen, S. (2000). "Theory of mind and autism: a fifteen year review," in Understanding Other Minds: Perspectives from Developmental Cognitive Neuroscience, eds S. BaronCohen, H. Tager-Flusberg, and D. J. Cohen (New York: Oxford University Press), 88-123.

Blasi, A., Fox, S., Everdell, N., Volein, A., Tucker, L., Csibra, G., Gibson, A., Hebden, J., Johnson, M. H., and Elwell, C. (2007). Investigation of depth dependent changes in cerebral haemodynamics during face perception in infants. Phys. Med. Biol. 52, 6849-6864.

Csibra, G. (2010). Recognizing communicative intentions in infancy. Mind Lang. 25, 141-168.

Davidson, R., and Fox, N. (1982). Asymmetrical brain activity discriminates between positive and negative affective stimuli in human infants. Science 218, 1235-1237.

Farroni, T., Csibra, G., Simion, F., and Johnson, M. H. (2002). Eye contact detection in humans from birth. Proc. Natl. Acad. Sci. U.S.A. 99, 9602-9605.

Farroni, T., Johnson, M. H., Menon, E., Zulian, L., Faraguna, D., and Csibra, G. (2005). Newborns' preference for face-relevant stimuli: effects of con-

trast polarity. Proc. Natl. Acad. Sci. 50, 531-534.

\section{ACKNOWLEDGMENTS}

Tobias Grossmann was supported by a Sir Henry Wellcome Fellowship awarded by the Wellcome Trust (082659/Z/07/Z). E. Parise was supported by a Humboldt Research Fellowship, Germany, and by a grant from Calabria Region, Italy. Thanks to Daniela Danz for managing the names' database and helping with testing. The work was conducted at the Max Planck Institute for Human Cognitive and Brain Sciences.

metrical frontal cortical activity. Psychophysiology 40, 838-848. U.S.A. 102, 17245-17250.

Fox, N. A. (1991). If it's not left, it's right: electroencephalogram asymmetry and development of emotion. Am. Psychol. 46, 863-872.

Frith, C. D., and Frith, U. (1999). Interacting minds - a biological basis. Science 286, 1692-1695.

Frith, C. D., and Frith, U. (2006). The neural basis of mentalizing. Neuron

Grossmann, T., and Farroni, T. (2009). "Decoding social signals in the infant brain: a look at eye gaze perception," in Handbook of Developmental Social Neuroscience, eds M. de Haan and M. Gunnar (New York, NY: Guilford Press), 87-106.

Grossmann, T., and Johnson, M. H. (2010). Selective prefrontal cortex responses to joint attention in early infancy. Biol. Lett. 6, 540-543.

Grossmann, T., Johnson, M. H., LloydFox, S., Blasi, A., Deligianni, F., Elwell, C., and Csibra, G. (2008). Early cortical specialization for face-to-face communication in human infants. Proc. R. Soc. Lond. B Biol. Sci. 275, 2803-2811.

Grossmann, T., Oberecker, R., Koch, S. P., and Friederici,A.D. (2010). The developmental origins of voice processing in the human brain. Neuron 65, 852-858.

Harmon-Jones, E. (2003). Clarifying the emotive functions of asym-
Johnson, M. H. (2005). Subcortical face processing. Nat. Rev. Neurosci. 6, 766-774.

Kampe, K. K. W., Frith, C. D., and Frith, U. (2003). "Hey John": signals conveying communicative intention toward the self activate brain regions associated with "mentalizing," regardless of modality. J. Neurosci. 23, 5258-5263.

Lloyd-Fox, S., Blasi, A., and Elwell, C. E. (2010). Illuminating the developing brain: the past, present and future of functional near infrared spectroscopy. Neurosci. Biobehav. Rev. 34, 269-284.

Mandel, D. R., Jusczyk, P. W., and Pisoni, D. B. (1995). Infants' recognition of the sound patterns of their own names. Psychol. Sci. 6, 314-317.

Moray, N. (1959). Attention in dichotic listening: affective cues and the influence of instructions. Q. J. Exp. Psychol. $11,56-60$.

Osterling, J.A., Dawson, G., and Munson, J. A. (2002). Early recognition of 1-yearold infants with autism spectrum disorder versus mental retardation. Dev. Psychopathol. 14, 239-251.

Senju, A., and Johnson, M. H. (2009). The eye contact effect: mechanisms and development. Trends Cogn. Sci. 13, 127-134.

Sperber, D., and Wilson, D. (1995). Relevance: Communication and Cognition, 2nd Edn. Oxford: Blackwell.
Strangman, G., Culver, J. P., Thompson, J. H., and Boas, D. A. (2002). A quantitative comparison of simultaneous BOLD fMRI and NIRS recordings during functional brain activation. NeuroImage 17, 719-731.

von Grünau, M., and Anston, C. (1995). The detection of gaze direction: a stare-in-the-crowd effect. Perception 24, 1297-1313.

Woodward, A. L. (2009). Infants' grasp of others' intentions. Curr. Dir. Psychol. Sci. 18, 53-57.

Conflict of Interest Statement: The authors declare that the research was conducted in the absence of any commercial or financial relationships that could be construed as a potential conflict of interest.

Received: 16 May 2010; paperpending published: 16 May 2010; accepted: 01 October 2010; published online: 25 October 2010. Citation: Grossmann T, Parise E and Friederici AD (2010). The detection of communicative signals directed at the self in infant prefrontal cortex. Front. Hum. Neurosci. 4:201. doi: 10.3389/ fnhum.2010.00201

Copyright (C) 2010 Grossmann, Parise and Friederici. This is an open-access article subject to an exclusive license agreement between the authors and the Frontiers Research Foundation, which permits unrestricted use, distribution, and reproduction in any medium, provided the original authors and source are credited. 